\title{
ULTRABRIGHT SYNCHROTRON SOURCE ELETTRA: FIRST PERIOD OF OPERATION
}

\author{
G. Margaritondo ${ }^{a, b}$, A. Savoia ${ }^{b}$, S. BernstofF ${ }^{b}$, M. Bertolo $^{b}$,

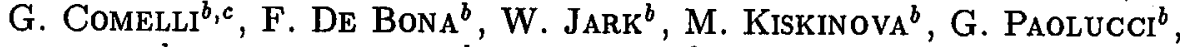 \\ K. Prince ${ }^{b}$, A. Santaniello ${ }^{b}$, G. Tromba ${ }^{b}$, R. WalkeR ${ }^{b}$ and R. Rosei ${ }^{b}, c$ \\ anstitut de Physique Appliquée, Ecole Polytechnique Fédérale \\ 1015 Lausanne, Switzerland \\ ${ }^{b}$ Sincrotrone Trieste SCpA, Trieste, Italy \\ ${ }^{c}$ Dipartimento di Fisica, Università di Trieste, Via A. Valerio 2, 34127 Trieste, Italy
}

ELETTRA, the brightest source of soft-X-ray in Europe and one of the two brightest in the world, is officially operating as a user facility since the summer of 1995 . The source and the operating beam lines have reached and surpassed the design performances. The impact of such performances is already felt in experiments: new applications are possible which were very difficult or quite impossible with the previous generation of sources. We present a few specific results to illustrate this point, and some considerations on the future development of the facility - primarily in view of its large and rapidly growing international use.

PACS numbers: 41.60.Ap, 79.60.-i

\section{ELETTRA as a working reality}

Since the fall of 1993, ELETTRA has operated as the brightest source of soft-X-rays in Europe [1]. The general philosophy of synchrotron facilities of its (third) generation is to bring the source brightness [2] to unprecedented level by a better control of the electron beam geometry and also by extensively using the insertion devices known as undulators. In emphasizing brightness, this philosophy marked a radical departure from the effort concentrated on the increase in the electron beam current and therefore in the overall emitted flux which had dominated the previous generations.

Contrary to the flux, the brightness also takes into account the geometrical characteristics of the beam: size and angular divergence [2]. The relatively recent emphasis on brightness is justified by arguing that the beam geometry plays a fundamental role in most synchrotron-light applications. This point, however, has been the subject of debate: some authors emphasized the relative merits of parameters other than brightness, such as - indeed - flux, or lifetime. 
The recent results from ELETTRA enable us to tackle this issue. ELETTRA worked informally as a user facility starting a few weeks after its first commissioning [1]. In the summer of 1995 , it was officially opened to users, who access its beam lines through a peer-review process based on merit. The data production already makes it possible to assess the impact of the ELETTRA brightness on real experiments.

The assessment is quite clear: the high brightness levels (up to nearly $10^{19}$ photons $/\left(\mathrm{mm}^{2} \mathrm{mrad}^{2} \mathrm{sec} ; 0.1 \%\right.$ bandwidth $)$ - see Table I for a summary of other ELETTRA parameters) make a huge difference in most experiments, creating entirely new research opportunities. In this short overview, we will present some examples that support this conclusion.

TABLE I

Main parameters of ELETTRA.

\begin{tabular}{l|l}
\hline \hline Electron beam energy: & $\begin{array}{l}1.5 \text { or } 2.0 \mathrm{GeV} \\
\text { (maximum achieved } 2.3 \mathrm{GeV} \text { ) }\end{array}$ \\
\hline Maximum beam current: & $530 \mathrm{~mA}$ \\
\hline Maximum brightness: & $\begin{array}{l}8 \times 10^{18} \text { photons } \mathrm{s}^{-1} \mathrm{mrad}^{-2} \mathrm{~mm}^{-2} \\
(0.1 \% \text { band width) } \\
\text { (at } 2 \mathrm{GeV} \text { and } 400 \mathrm{~mA})\end{array}$ \\
\hline Lifetime (relaxed optics): & $\begin{array}{l}30 \mathrm{~h} \text { at } 250 \mathrm{~mA} \\
>40 \mathrm{~h} \text { at } 100 \mathrm{~mA}\end{array}$ \\
\hline Reliability: & $\approx 93 \%$
\end{tabular}

\section{Ultrahigh brightness in action}

A good way to assess the impact of the new brightness levels is to analyze the evolution of established synchrotron spectroscopy techniques [2]. The benchmark in our case is photoelectron spectroscopy [2], whose technical improvements in the past three decades closely tracked those of the synchrotron sources: from angular resolution with the first-generation facilities to preliminary steps into lateral resolution with the best second-generation facilities.

ELETTRA brings photoemission techniques to top performance levels as far as energy, angle, space and time resolutions are concerned, while keeping the signal at high levels. Consider first the VUV Photoemission beam line, which is designed to enhance flux and energy resolution. Figure 1 shows one of the first results, the high-resolution spectrum of the $3 d_{5 / 2}$ core level of the (110) face of rhodium by Zacchigna et al. [3].

The left-hand peak is due to the emission from the bulk and the right-hand peak to surface emission. A least-square fit (dashed curves) also reveals a second contribution to the left-hand peak, due to the second layer of atoms. The high-resolution spectra make it possible to detect a difference in line width between the two components: the surface-emission peak is noticeably broader. Fur- 


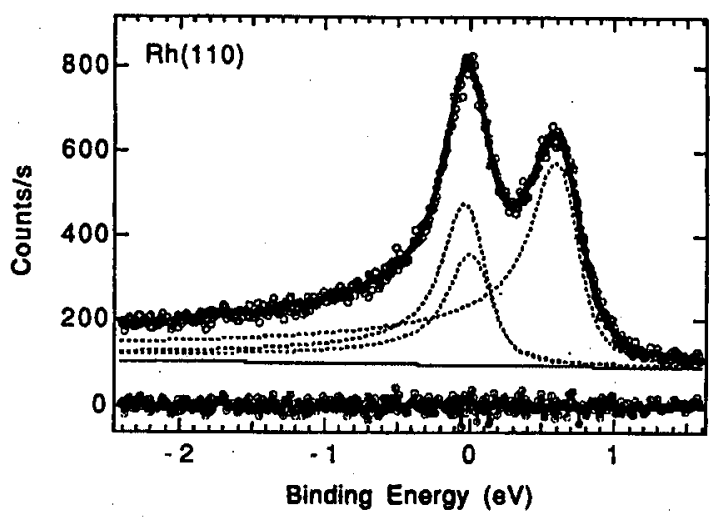

Fig. 1. High-resolution core level photoemission spectrum of $\mathrm{Rh} 3 d_{5 / 2}$, from the ELETTRA VUV Photoemission beam line. Data from Ref. [3].

thermore, a data analysis reveals that the surface peak is more asymmetric, i.e., its singularity index is higher.

The higher singularity index indicates that each surface atom is surrounded by a higher density of screening charge at the Fermi level. The larger width of the surface peak is due to the Lorentzian component of the total line shape: a larger Lorentzian indicates a shorter lifetime, and this again indicates a higher charge density projected on the core of the surface atoms. However, in this case it is not just the Fermi level density, but the total charge.

Measurements of line shapes of deep core levels like those of Fig. 1 are very difficult or quite impossible with second-generation sources due to limited resolution and flux. Figure 1, therefore, is a good example of the ELETTRA brightness at work.

Figure 2 shows a similarly good example in the case of spatial resolution, with data taken by Kolmakov et al. [4] on the ESCA Microscopy beam line, which uses Fresnel zone-plate focusing to achieve high lateral resolution in photoemission spectroscopy. With 0.2 micron lateral resolution, this facility can attack entirely new problems on a microscopic scale, with the full analytical power of "macroscopic" photoemission spectroscopy.

The beam line is an ideal tool to study heterogeneous interfaces: the results of Fig. 2 reveal the influence of the surface microstructure on chemical reactivity at a laterally heterogeneous metal-semiconductor interface. Figure $2 \mathrm{a}$ shows photoelectron micrographs of the investigated system, which was obtained by first depositing and annealing $\mathrm{Au}$ on $\mathrm{Si}(111)$ and then again depositing $\mathrm{Ag}$. The Au deposition results in coexisting two-dimensional (2D) and 3-dimensional (3D) phases, with the latter consisting of submicron Au particles. The results elucidate the influence of the two phases on the overall interface formation process.

The micrographs of Fig. 2a were taken by tuning the collected photoelectron energy to the $\mathrm{Au} 4 f_{7 / 2}$, Si $2 p$ and $\mathrm{Ag} 3 d_{3 / 2}$ core level peaks and reveal the spatial distribution of the corresponding elements. The images immediately reveal the different substrate-overlayer interaction for the two phases: the Au-rich 3D islands 
(a)
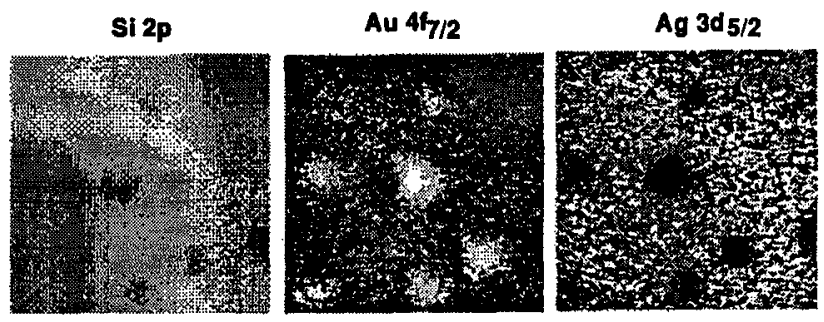

(b)

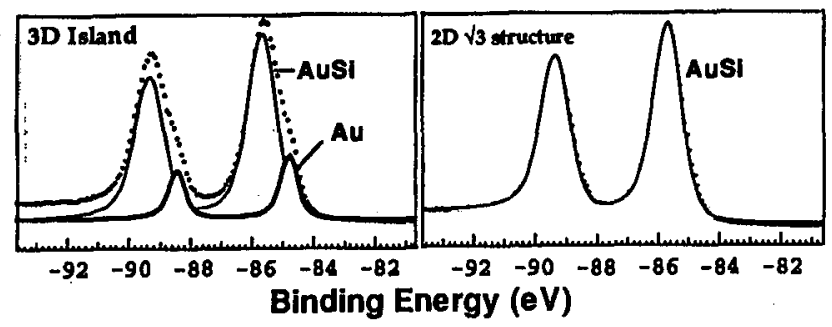

Fig. 2. (a) Photoelectron micrographs showing the lateral distribution of $\mathrm{Si}, \mathrm{Au}$ and $\mathrm{Ag}$ for an Ag-covered $\mathrm{Si}(111)+\mathrm{Au}$ substrate. The system includes two-dimensional (2D) and three-dimensional (3D) phases. (b) Au $4 f_{7 / 2}$ spectra for the 3D and 2D phases. Data from the ESCA Microscopy beam line, Ref. [4].

contain less $\mathrm{Ag}$ and $\mathrm{Si}$ than the $2 \mathrm{D}$ phase. Furthermore, photoemission spectra taken in small areas reveal the differences in chemical status between the two phases. In fact, the space-resolved $\mathrm{Au} 4 f$ spectra of Fig. $2 \mathrm{~b}$ show that the 3D particles contain both $\mathrm{AuSi}$ and metallic gold, whereas the $2 \mathrm{D} \sqrt{3}-\mathrm{Ag}+\mathrm{Au} / \mathrm{Si}$ phase contains only AuSi. The local Au chemical status determines the kinetics of the $\mathrm{Ag}-\mathrm{Au}$ intermixing: the presence of metallic $\mathrm{Au}$ explains the higher reactivity of the $3 \mathrm{D}$ particles [4].

Figure 3 brings us from spatial resolution to time resolution, which is the specialty of the SuperESCA beam line [5]. This facility can take spectra with excellent energy resolution and high signal level in a few seconds, soon to be pushed to lower levels by a new detection electronics. In this way, it can take fast sequences of spectra - real "chemical movies" of surface chemical reactions.

The data of Fig. 3 (Ref. [5]) reveal the time evolution of the O $1 s$ spectrum while a $\operatorname{Rh}(110)$ surface is exposed to an oxygen partial pressure of $5 \times 10^{-9} \mathrm{mbar}$ at room temperature. Only a subset of the complete 80-spectra set is shown, each measured in $12 \mathrm{sec}$ and able to track coverage variations of $c a .0 .02$ monolayers.

A species with $529.65 \pm 0.05 \mathrm{eV}$ binding energy appears first, and is the only one up to a coverage of 0.35 monolayers. Then, the signal from the first species decreases and a second species appears at $530.25 \pm 0.05 \mathrm{eV}$ binding energy; after 0.8 monolayer coverage, only the second species is seen.

These results are interpreted in terms of adsorption in the long bridge site followed by adsorption in the three-fold sites of the (110) surface. The dominance 


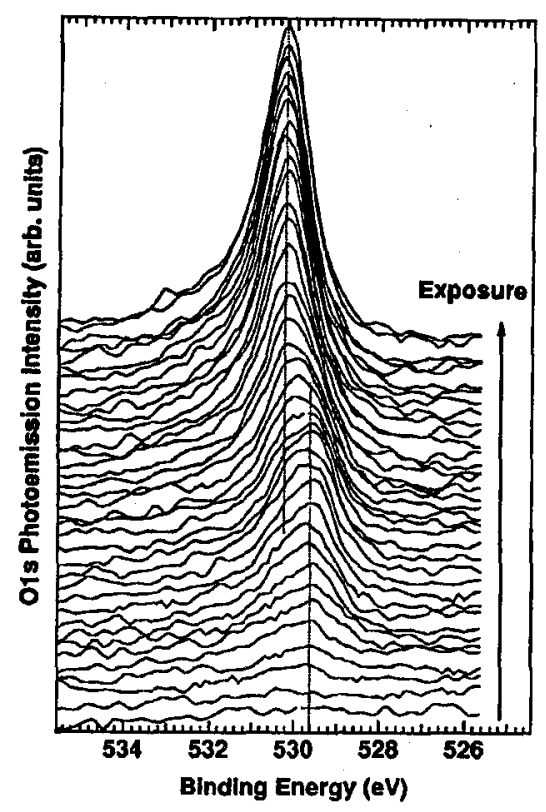

Fig. 3. A series of time-resolved photoemission O $1 s$ spectra taken on the SuperESCA beam line (Ref. [5]) during the adsorption of oxygen on $\mathrm{Rh}(110)$ at room temperature.

of the second site at high coverage is due to $\mathrm{O}-\mathrm{O}$ interaction which becomes important only beyond 0.35 monolayer coverage. Oxygen atoms originally adsorbed in the long bridge sites are displaced to three-fold sites.

These results are just examples of the many features that can be explored with time-resolved synchrotron photoelectron spectroscopy. One can, in general, analyze the interplay between adsorption rate and the changes occurring at the surface during adsorption: adsorbate coverage, occupation and changes from one adsorption site to another. Note that alternate approaches are quite difficult to use for problems like those of Fig. 3: vibrational techniques are not easily applied to atomic adsorbates, and other experiments, like sticking-coefficient measurements with molecular beams, give only integrated (non-species-resolved) information.

ELETTRA is optimized for soft-X-ray emission, therefore spectrally complementary to the high-energy facilities of the third generation - such as ESRF in Grenoble and APS at Argonne. Nevertheless, ELETTRA does reach excellent performances in some of the techniques that use higher-energy photons than the previous examples. Figure 4 shows a nice case: the solution of a structural problem with crystallographic techniques, which was completed by Mesters et al. [6] on the ELETTRA X-ray Diffraction beam line.

The ribbon diagram in the figure refers to the structural aspects of the interaction of the elongation factor Tu (EF-Tu) with aminoacyl-tRNA (aa-tRNA). EF-Tu is one of the most versatile biological macromolecules so far analyzed; it binds aa-tRNA, components of the ribosome, guanine nucleotides, a magnesium ion, and a nucleotide exchange factor, EF-Ts. The three domains of EF-Tu undergo 


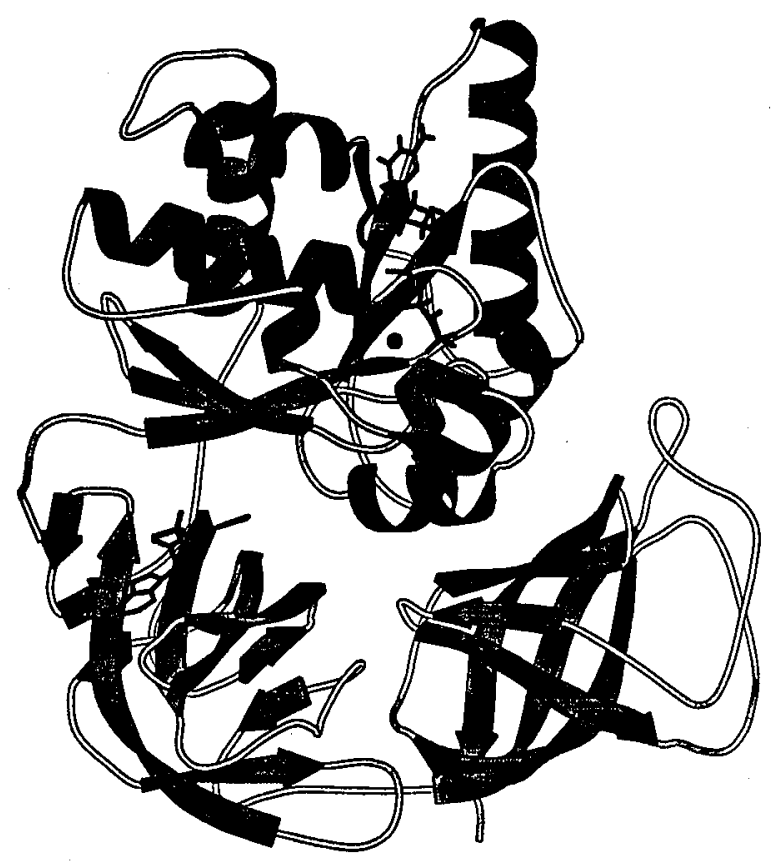

Fig. 4. Ribbon diagram showing the main-chain structure of the active form of elongation factor $\mathrm{Tu}$, in complex with guanosine triphosphate and with anthraniloyl adenosine-6'-monophosphate (ant-AMP). Note the ant-AMP (the small dark-grey molecule on the left) in the cleft. Data partly from the ELETTRA X-ray Diffraction beam line, see Ref. [6].

major mutual rearrangements upon the transition from the active, GTP-bound form (GTP=guanosintriphosphate) of the protein to its inactive GDP-bound form (GDP=guanosindiphosphate).

A deep cleft located between domains 1 and 2 of the GTP form is the binding site for aa-tRNA; this cleft is not present in EF-Tu*GDP, the complex of EF-Tu and GDP: The experiments solved the crystal structure of the ternary complex consisting of EF-Tu, GppNHp and anthraniloyl adenosine- $6^{\prime}$-monophosphate (ant-AMP). The last one is a model compound for the aminoacylated $3^{\prime}$ terminal adenine of tRNA. This small molecule binds to EF-Tu*GTP (but not to EF-Tu*GDP) and exhibits many of the effects of the whole aa-tRNA.

Figure 4 shows indeed the ant-AMP molecule (the small dark-gray molecule on the left-hand side)) in the cleft. Overall, the ribbon diagram illustrates the main-chain structure of the active form of EF-Tu, in complex with ant-AMP and with guanosine triphosphate (the small black molecule near the top). The analog for the aminoacylated $3^{\prime}$ terminal adenine of tRNA is seen to bind to domain 2 but interacting with domain 1 as well.

Also operating in the higher-photon-energy side of the ELETTRA beam lines is the latest addition to the family of operating facilities: the Austrian Academy of Sciences Small-Angle X-ray Scattering (SAXS) beam line [7]. This apparatus can 
perform wide and small angle measurements in: gels, liquid crystals, (bio)polymers, amorphous materials, etc. and can operate both on weakly and strongly absorbing samples. The beam line obtains photons from the same wiggler as the X-ray Diffraction facility, works at photon energies up to $16 \mathrm{keV}$, and is equipped with a water-cooled double-crystal monochromator.

Since early 1996, SAXS is a working reality. Figure $5 \mathrm{a}$ shows $[7,8]$ its measured vertical and horizontal focal spot sizes: the full width at half maximum (FWHM) is $1.5 \mathrm{~mm}$ horizontal and $0.6 \mathrm{~mm}$ vertical, to be compared with calculated values of 1.2 and $0.7 \mathrm{~mm}$. Figure $5 \mathrm{c}$ is a historically relevant result, crowning the work of the beam line constructors (under the leadership of Peter Laggner): the first spectrum of SAXS [8].
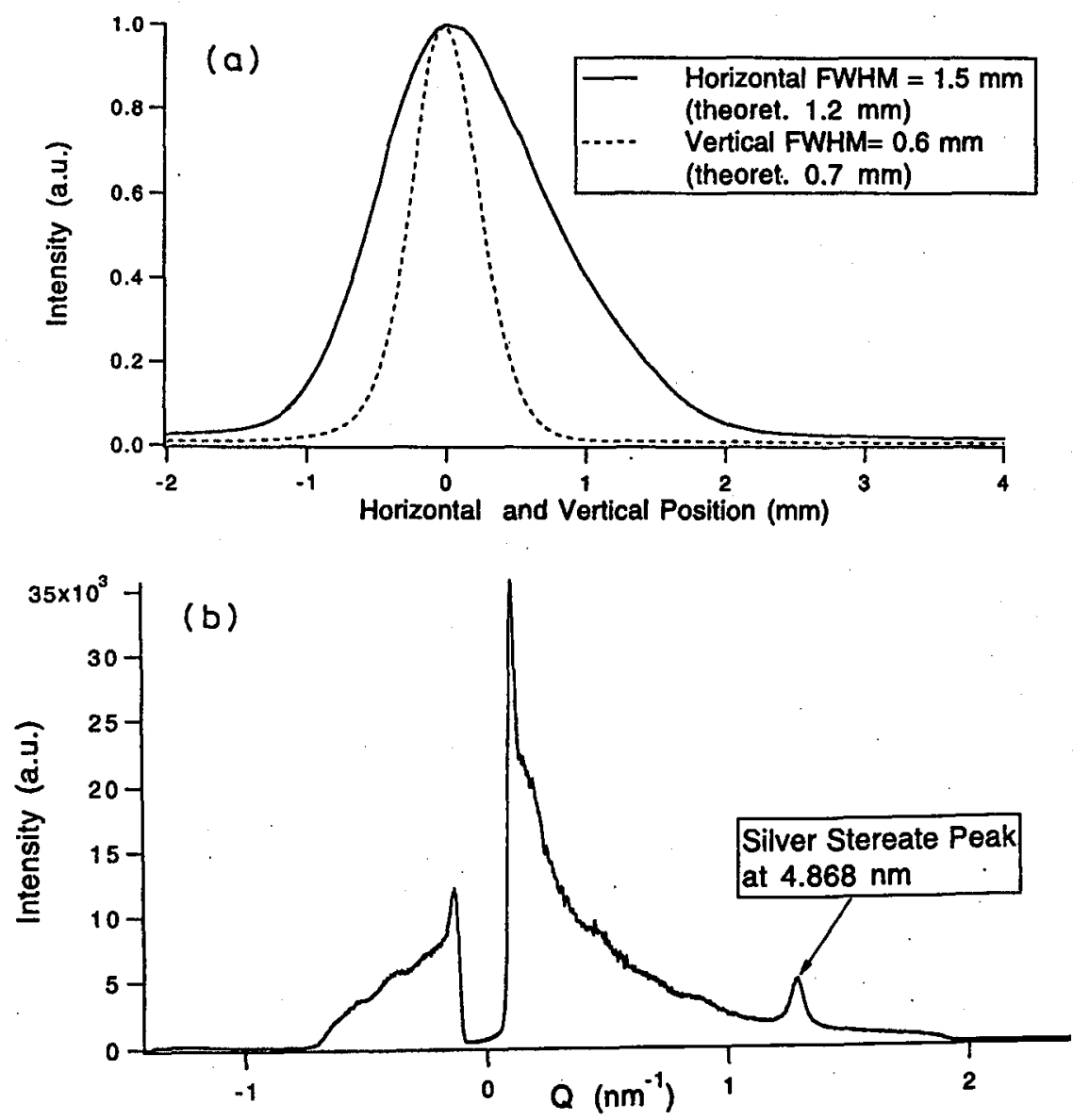

Fig. 5. (a) Measured horizontal and vertical spot sizes of the Austrian Academy of Sciences SAXS beam line on ELETTRA; (b) the first spectrum from the same beam line taken on 14.12 .95 at 15:27 o'clock, data from Refs. [7, 8]. 


\section{Even brighter future}

ELETTRA now produces every week results of class comparable to those of Figs. 1-5. This fact is becoming known to scientists throughout the world and the results are a mixed blessing. In fact, the demand for beam time creates an average oversubscription that reached early in 1996 the $329 \%$ level (with a peak of almost $1000 \%$ for a specific beam line). This is a very severe problem as far as the overall beam time allocation is concerned.

The ELETTRA staff is trying to alleviate this problem with a broad beam line development program, which should add 6-8 new facilities between 1996 and mid-1997 to the five that are already operating, discussed above. Some of the new beam lines enhance the capabilities in areas already covered by operating facilities: Scanning Spectromicroscopy (SuperMAXIMUM) will complement ESCA Microscopy by using Schwarzschild objectives rather than Fresnel zone plates to focus lower-energy photons; ALOISA will work on surface X-ray diffraction, multi-particle coincidence experiments and photoelectron diffraction; the Gas Phase Photoemission beam line will serve the traditionally strong atomic and molecular research community in Italy as well as their European colleagues. These undulator-based facilities will be accompanied by two advanced bending-magnet facilities: the Materials Science Beam line of the Czech Academy of Sciences, and the Beam line of Slovenian Scientists (BOSS).

Other beam lines go into entirely new directions, not covered by the present facilities. Such is the case of the Microfabrication (LIGA) beam line, dedicated to the lithography-galvanoplastic-molding process for the fabrication of microscopic mechanical parts. And of SYRMEP, a beam line which has for objective the early detection of breast cancer in the female population, and specifically to increase resolution and contrast by using monochromatic X-rays (optimized for each single patient) and by reducing scattering with the use of a laminar beam. SYRMEP, which will include a new digital Si detector, is in an advanced commissioning stage: the first X-ray beam just went through the line. This project is a joint venture of the University of Trieste, of the INFN-Trieste and of ELETTRA (project leader: E. Castelli).

Finally, there is a quite ambitious project in the domain of circular polarization: a project supported by European funds and based on the development of an elliptical wiggler optimized for $5-1500 \mathrm{eV}$. The main parameters have just been decided. The wiggler period will be $200 \mathrm{~mm}$, and the device will include 30 periods. The vertical magnetic field will be $0.5 \mathrm{~T}$, giving a vertical $k$-parameter of 9.3 ; the horizontal values will be $0.08 \mathrm{~T}$ and 1.5 . The critical energy will be $1.3 \mathrm{keV}$.

More in the future, the ELETTRA staff is planning the addition of a free-electron-laser project to the other facilities. This plan, code-named FERMI project, is explained in detail in Ref. [9].

With these new facilities (see Table II for a summary) and with the additional ones that are already foreseen - notably the University of Trieste EXAFS facility - ELETTRA will be able to serve an international community. Built with Italian resources by an international team, ELETTRA is being used by an increasing number of non-Italian scientists, who already constitute the majority of 
TABLE II

Status of the ELETTRA beam lines operating or under development (spring 1996).

\begin{tabular}{l|l|l}
\hline \hline Beam line & Type of source & Status \\
\hline SuperESCA & undulator & operating \\
\hline ESCA Microscopy & undulator & operating \\
\hline Vacuum Ultraviolet Photoemission & undulator & operating \\
\hline X-ray Diffraction & wiggler & operating \\
\hline $\begin{array}{l}\text { Small-Angle X-ray Scattering } \\
\text { (Austria) }\end{array}$ & wiggler & $\begin{array}{l}\text { operating } \\
\text { (final } \\
\text { commissioning) }\end{array}$ \\
\hline Gas Phase Photoemission & undulator & $\begin{array}{l}\text { under } \\
\text { construction }\end{array}$ \\
\hline Surface Diffraction (ALOISA) & undulator & $\begin{array}{l}\text { under } \\
\text { construction }\end{array}$ \\
\hline Mammography (SYRMEP) & bending magnet & $\begin{array}{l}\text { final } \\
\text { commissioning }\end{array}$ \\
\hline Microfabrication (LIGA) & bending magnet & $\begin{array}{l}\text { under } \\
\text { construction }\end{array}$ \\
\hline $\begin{array}{l}\text { Circular Polarization } \\
\text { Scanning Spectromicroscopy } \\
\text { (SuperMAXIMUM) }\end{array}$ & undulator & $\begin{array}{l}\text { under } \\
\text { construction }\end{array}$ \\
\hline $\begin{array}{l}\text { Materials Science } \\
\text { (Czech Republic) }\end{array}$ & bending magnet & $\begin{array}{l}\text { initial } \\
\text { construction }\end{array}$ \\
\hline EXSS & bending magnet & $\begin{array}{l}\text { initial } \\
\text { construction }\end{array}$ \\
\hline EXS & bending magnet & planning \\
\end{tabular}

the users. This de facto internationalization is quite important for scientists in Central-Eastern Europe: users from Poland, Austria, Slovenia, Croatia, the Czech Republic, Switzerland, Hungary, Slovakia and other countries find it quite easy to take advantage of the brightest soft-X-ray source in the European continent [10]. ELETTRA is thus standing up to the words that have been recently used to describe its changing character; "Built by Italy, Open to the World".

\section{Acknowledgments}

The results used as examples in this overview were produced in collaboration with many scientists, including all authors of Refs. [3-9]. Support for ELETTRA is provided by the Sincrotrone Trieste SCpA, by the European Commission and 
by the Austrian Academy of Sciences. One of the authors (G.M.) acknowledges support by the Fonds National Suisse de la Recherche Scientifique and by the Ecole Polytechnique Fédérale de Lausanne.

\section{References}

[1] A. Abrami, D. Alfe, S. Antonini, M. Bernardini, M. Bertolo, C.J. Bocchetta, D. Bulfone, F. Cargnello, F. Daclon, S. Di Fonzo, S. Fontana, A. Galimberti, M. Giannini, W. Jark, A. Massarotti, F. Mazzolini, M. Puglisi, R. Richter, A. Rindi, R. Rosei, C. Rubbia, D. Tommasini, A. Savoia, G. Viani, R.P. Walker, A. Wrulich, C. Coluzza, Tiziana dell'Orto, F. Gozzo, G. Margaritondo, G. De Stasio, P. Perfetti, M. Gentili, M.T. Ciotti, D. Mercanti, Nucl. Instrum. Meth. A 349, 609 (1994).

[2] G. Margaritondo, Introduction to Synchrotron Radiation, Oxford University Press, New York 1988; G. Margaritondo, J. Synchr. Rad. 2, 148 (1995) and the references therein; Riv. Nuovo Cimento 18, 1 (1995) and the references therein.

[3] M. Zacchigna, C. Astaldi, K.C. Prince, M. Sastry, C. Comicioli, M. Evans, R. Rosei, C. Quaresima, C. Ottaviani, C. Crotti, M. Matteucci, P. Perfetti, to be published.

[4] A. Kolmakov, L. Kovac, M. Günther, L. Casalis, L. Gregoratti, M. Marsi, M. Kiskinova, to be published.

[5] A. Baraldi, M. Barnaba, B. Brena, D. Cocco, G. Comelli, S. Lizzit, G. Paolucci, R. Rosei, J. Electron Spectr. 76, 145 (1995); G. Comelli, A. Baraldi, S. Lizzit, G. Paolucci, R. Rosei, to be published.

[6] J. Mesters, W. Webster, R. Hilgenfeld, L. Olivi, A. Savoia, B. Nawrot, M. Sprinzl, to be published.

[7] P. Laggner, H. Amenitsch, S. Bernstoff, private communication.

[8] H. Amenitsch. S. Bernstoff, M. Chemlouil, M. Krieghbaum, H. Mio, P. Laggner, to be published.

[9] R.P. Walker, D. Bulfone, F. Cargnello, M. Castellano, F. Cevenini, F. Ciocci, A. Cutolo, G. D'Auria, F. Daclon, G. Dattoli, A. DeAngelis, A. Dipace, A. Doria, M. Ferianis, G.P. Gallerano, F. Garosi, L. Gianessi, M. Giannini, E. Giovenale, G. Margaritondo, A. Massarotti, L. Mezi, P.L. Otta viani, P. Patteri, A. Renieri, A. Rindi, R. Rosei, C. Rubbia, E. Sabia, A. Segreto, S. Tazzari, F. Tazzioli, A. Torre, R. Visintini, A. Wrulich, D. Zangrando, Nucl. Instrum. Meth. (in press) and the references therein.

[10] Potential users can find additional information on ELETTRA and the user procedures by accessing its homepage (http:/www.elettra.trieste.it). 